\title{
Synthesis and Catalytic Performances of a Novel Zn-MOF Catalyst Bearing Nickel Chelating Diimine Carboxylate Ligands for Ethylene Oligomerization
}

\author{
Suyan Liu, ${ }^{1}$ Ying Zhang, ${ }^{1}$ Quan Huo, ${ }^{2}$ Sasa He, ${ }^{1}$ and Yang Han ${ }^{1}$ \\ ${ }^{1}$ The State Key Laboratory of Heavy Oil Processing, Department of Materials Science and Engineering, China University of \\ Petroleum (Beijing), Changping District, Beijing 102249, China \\ ${ }^{2}$ Hebei Key Laboratory of Applied Chemistry, College of Environmental and Chemical Engineering, Yanshan University, \\ Qinhuangdao, Hebei 066004, China
}

Correspondence should be addressed to Ying Zhang; y.zhang@cup.edu.cn and Quan Huo; quanhuo@ysu.edu.cn

Received 31 July 2014; Accepted 20 August 2014

Academic Editor: Xinqing Chen

Copyright (C) 2015 Suyan Liu et al. This is an open access article distributed under the Creative Commons Attribution License, which permits unrestricted use, distribution, and reproduction in any medium, provided the original work is properly cited.

A novel $\mathrm{Zn}-\mathrm{MOF}\left[\mathrm{Zn}_{3}(\mathrm{OH})_{2} \mathrm{~L}_{2}\right]$ was synthesized from dicarboxylate ligands with diimine groups (1,4-bis $\left(4-\mathrm{CO}_{2} \mathrm{HC}_{6} \mathrm{H}_{4}\right)-2,3-$ dimethyl-1,4-diazabutadiene). The physicochemical properties of the material were characterized by a series of technologies including XRD, SEM, and ICP. In order to adapt to the ethylene oligomerization process, a catalyst $\left[\mathrm{Zn}_{3}(\mathrm{OH})_{2}\left(\mathrm{~L}_{1}{ }^{\mathrm{Ni}}\right)_{2}\right](\mathrm{denoted}$ as Cat.A) possessing active $\mathrm{Ni}^{2+}$ centers was prepared by a postsynthetic treatment method using dichloride nickel as a nickel source in this work. For comparison, $\alpha$-diimine ligands with/without dicarboxylic acid groups reacted with dichloride nickel to obtain homogenous Cat.B and Cat.C, respectively. The effects of reaction parameters, including $n(\mathrm{Al}) / n(\mathrm{Ni})$, temperature, and pressure on the oligomerization activities and oligomers distribution were investigated. The results demonstrated that all of catalysts used with diethylaluminum chloride were active for the ethylene oligomerization. Among them, Cat.A and Cat.B showed higher catalytic activities and higher selectivities to low-carbon $\alpha$-olefins at atmospheric pressure. The Cat.A exhibited the optimal catalytic activity $\left[6.7 \times 10^{5} \mathrm{~g} /(\mathrm{mol} \cdot \mathrm{Ni} \cdot \mathrm{h} \cdot \mathrm{atm})\right]$ for $\mathrm{C} 4(91.8 \%)$ under the conditions of $\mathrm{Al} / \mathrm{Ni}=1500, P=1.0 \mathrm{~atm}, \mathrm{~T}=20^{\circ} \mathrm{C}$. In addition, Cat.A and Cat.B presented large amount of ethylene polymer, while Cat.C had a higher catalytic activity of ethylene oligomerization at high pressure.

\section{Introduction}

Low-carbon linear $\alpha$-olefins are extensively used as comonomer of linear low-density polyethylene, plasticizers, and synthetic lubricants $[1,2]$. Among technologies of producing low-carbon linear $\alpha$-olefins, ethylene oligomerization is a major industrial process. Today, the well-known industrial processes of ethylene oligomerization include Shell Higher Olefin Process (SHOP) employing homogeneous nickel catalysts bearing $[\mathrm{P}, \mathrm{O}]$ ligands and Philips ethylene selective trimerization or tetramerization processes using homogeneous chromium catalysts [3, 4]. Although a lot of research efforts focus on homogenous oligomerization catalysts, the process has a serious drawback of separation of oligomerization products from catalysts and solvent mixtures. The added separation process will lead to the significant increase of operation costs and environmental pollution. With respect to the green chemistry principles, heterogeneous catalysis is an environmentally friendly alternative to the traditional homogenous process. Heterogeneous catalysts using porous solids (MCM-36, MCM-22, [5] MCM41 [6]) as supporting materials exhibit good catalysis performances for the ethylene oligomerization. Unfortunately, characteristic results show that anchored active species are not evenly located within pore channels. As a result, a rapid enrichment of polymeric waxes occurs in the part; thus the selectivity to low-carbon linear $\alpha$-olefins has been seriously affected. This phenomenon is commonly encountered in ethylene oligomerization processes using porous solids as the supports of catalysts. Therefore, the development 
of new supporting strategy on porous materials is desirable.

In the past decade, metal-organic frameworks (MOFs) have attracted a great deal of attention [7]. Due to their outstanding features of tunable pore sizes, high specific surface areas, and the possibility to functionalize, they have been widely used in the processes of hydrogen storage [8, 9], gas separation [10], catalysis [11], sensing [12, 13], and drug delivery $[14,15]$. In particular, an important feature of MOFs as catalysts is their self-assembled metal active sites, which are uniformly distributed over porous frameworks. Recently, MOFs for ethylene oligomerization have come into notice by several research groups. Kyogoku and coworkers [16] have prepared metal-organic framework (MOF) compounds containing Ni-bipyridyl complex. The material used with diethyl aluminum chloride had high potential as a catalyst for the oligomerization of ethylene with high selectivity for linear butenes. Canivet and coworkers [17] have anchored a molecular nickel complex into a mesoporous metal-organic framework (Ni@(Fe)MIL-101) using the one-pot postfunctionalization method. It is generating a very active and reusable catalyst for the liquid-phase ethylene dimerization to selectively form 1-butene. Mlinar and coworkers [18] have reported two $\mathrm{Ni}^{2+}$-containing metalorganic frameworks with high concentrations of coordinatively unsaturated $\mathrm{Ni}^{2+}$ sites, which exhibit activity comparable to $\mathrm{Ni}^{2+}$-exchanged aluminosilicates but maintain high selectivity for linear oligomers. However, examples of metalorganic framework bearing metal-binding organic ligands as ethylene oligomerization catalysts compared with the beforeassembled metalloorganic ligands, particularly in liquidphase oligomerization reactions, are rare.

Here we are interested in assembling dicarboxylate ligands with diimine groups into MOF framework followed by nickel complexation with $\alpha$-diimine groups to realize the self-support of nickel active sites. The difference of catalytic performances of these self-supported nickel active sites in the ethylene oligomerization between nickel sites coordinated with corresponding unassembled ligands is of interest. For this purpose, $\alpha$-diimine ligand with dicarboxylic acid $\left(\mathrm{L}_{1}\right.$, Scheme 1) is synthesized according to literature [19]. Such ligand as a linker is connected by $\mathrm{Zn}^{\mathrm{II}}$ ions nodes to construct MOF framework. The catalyst active $\mathrm{Ni}^{2+}$ centers are generated by postsynthetic treatment of MOF with dichloride nickel to form $\left[\mathrm{Zn}_{3}(\mathrm{OH})_{2}\left(\mathrm{~L}_{1}{ }^{\mathrm{Ni}}\right)_{2}\right]$ (Cat.A). For comparison, $\alpha$-diimine ligands with dicarboxylic acid groups $\left(\mathrm{L}_{1}\right)$ and without dicarboxylic acid groups $\left(\mathrm{L}_{2}\right)$ react with dichloride nickel to obtain homogenous Cat.B and Cat.C [20]. All the three catalysts are employed in the ethylene oligomerization and their catalytic performance is investigated under various reaction conditions.

\section{Experimental}

\subsection{Material Preparations}

2.1.1. Preparation of $\mathrm{NiL}_{1} \mathrm{Cl}_{2}$ (Cat.B) and $\mathrm{NiL}_{2} \mathrm{Cl}_{2}$ (Cat.C) Catalysts. $\mathrm{NiL}_{2} \mathrm{Cl}_{2}$ catalyst was synthesized according to the method described in the literature [20]. Similarly, the mixture composed of aminobenzoic acid and 2,3-butanedione was stirred at room temperature by Schiff-Alkali condensation reaction to obtain ligand $\mathrm{L}_{1}$ [19]. The dichloride nickel catalysts Cat.B were obtained by stirring the ligand $\mathrm{L}_{1}$ (0.84 g, $2.6 \mathrm{mmol}), \mathrm{NiCl}_{2}$ (DME) $(0.57 \mathrm{~g}, 2.6 \mathrm{mmol})$ with THF $(30 \mathrm{~mL})$ at room temperature for $48 \mathrm{~h}$. Then, the powder was washed by ether for three times and dried to obtain the $\mathrm{NiL}_{1} \mathrm{Cl}_{2}$ catalyst.

\subsubsection{Preparation of $\left[\mathrm{Zn}_{3}(\mathrm{OH})_{2}\left(\mathrm{~L}_{1}{ }^{\mathrm{Ni}}\right)_{2}\right]$ (Cat.A) Catalyst.} $\left[\mathrm{Zn}_{3}(\mathrm{OH})_{2}\left(\mathrm{~L}_{1}{ }^{\mathrm{Ni}}\right)_{2}\right]$ catalyst was synthesized according to the method described in the literature and divided into two processes [21]. First, ligand $\mathrm{L}_{1}(0.97 \mathrm{~g}, 0.3 \mathrm{~mol})$ was added to ethanol solution and stirred at room temperature for 4 hours, then $\mathrm{Zn}\left(\mathrm{NO}_{3}\right)_{2} \cdot 6 \mathrm{H}_{2} \mathrm{O}$ was added to the mixture, dripping $1.5 \mathrm{~mL}$ triethylamine and stirred for $8 \mathrm{~h}$, and the power ( $\mathrm{Zn}-\mathrm{MOF})$ was collected by repeated centrifugation and thorough washing with distilled water and dried in vacuum at $60^{\circ} \mathrm{C}$ for $5 \mathrm{~h}$. Then, the mixture composed of $\mathrm{Zn}-\mathrm{MOF}$ (0.84 g, $2.6 \mathrm{mmol}$ ), $\mathrm{NiCl}_{2}$ (DME) (0.54 g, $2.5 \mathrm{mmol}$ ), and THF (30 mL) was stirred for $48 \mathrm{~h}$ to obtain powder. The powder was washed by ether for three times and dried. The obtained product is $\left[\mathrm{Zn}_{3}(\mathrm{OH})_{2}\left(\mathrm{~L}_{1}{ }^{\mathrm{Ni}}\right)_{2}\right]$ catalyst.

2.2. Characterization. The crystallinity and phase purity of the product were measured by means of XRD patterns, which were recorded in a Shimadzu X-6000 diffractometer in the 2 theta range of $1.3-30^{\circ}$ at a scan speed of $4^{\circ} / \mathrm{min}$ using $\mathrm{Cu} \mathrm{KR}$ radiation to determine the crystallinity. The photographs of samples were taken by scanning electron microscopy (SEM) analyses, which were performed using FEI-QUANTA 200F equipment. The chemical compositions of the samples were measured by inductively coupled plasma (ICP) technique on a Perkin-Elmer Optima 3300DV spectrometer.

\subsection{Ethylene Oligomerization}

2.3.1. Procedure for Atmospheric Pressure Ethylene Oligomerization and Polymerization. The as-prepared nickel catalyst was added to a fully dried Schlenk flask under nitrogen. The flask was backfilled three times with $\mathrm{N}_{2}$ and twice with $1 \mathrm{~atm}$ ethylene, and then charged with toluene and $\mathrm{AlEt}_{2} \mathrm{Cl}$ solution in turn. Under prescribed temperature, the reaction solution was vigorously stirred under 1 atm ethylene for the desired period. The polymerization reaction was quenched by addition of $10 \% \mathrm{HCl} / \mathrm{EtOH}$ solution. About $1.0 \mathrm{~mL}$ of organic solution was taken for GC analysis. The remained mixture was poured into $100 \mathrm{~mL}$ of ethanol to precipitate the polymer. The polymer was isolated via a filtration and dried at $60^{\circ} \mathrm{C}$ to constant weight in a vacuum oven. The activity of oligomer (Ao) or polymer (Ap) was calculated according to the following equation:

$$
\text { Ao }(\text { Ap })=\frac{\text { Yield }(g) / \text { catalyst }(\mathrm{mol})}{\text { Time }(h) \times \text { Pressure }(\text { atm })}
$$




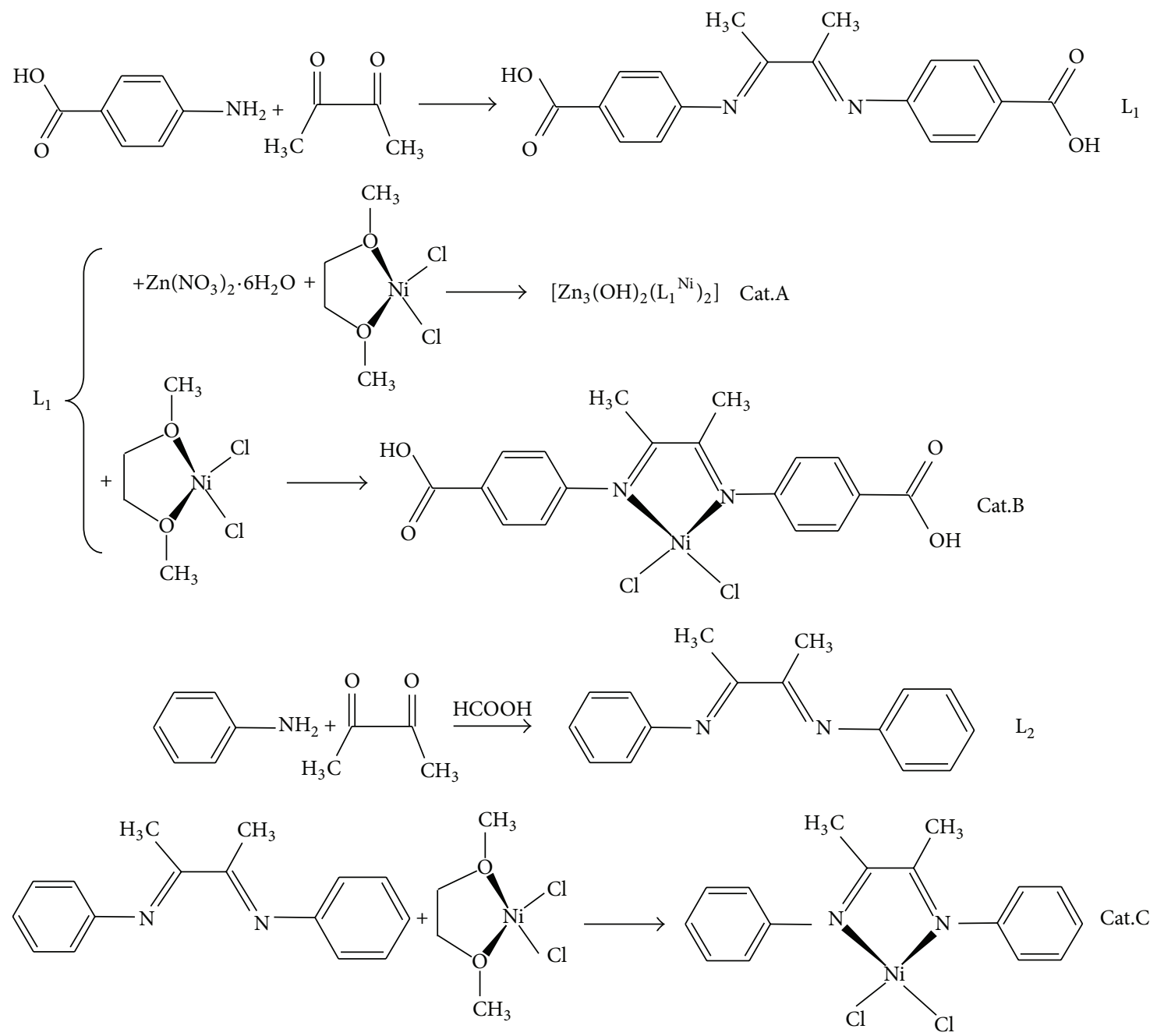

Scheme 1: Prepared processes of the three catalysts.

wherein the yield of oligomers was calculated by referencing with the mass of the solvent on the basis of the prerequisite that the mass of each fraction is approximately proportional to its integrated areas in the GC trace, and the yield of polymer was directly obtained by weighing polymer product with an electronic balance.

\subsubsection{Procedure for High-Pressure Ethylene Oligomerization} and Polymerization. High-pressure ethylene polymerization was performed in a stainless steel autoclave $(500 \mathrm{~mL}$ capacity) equipped through a solenoid clave for continuous feeding of ethylene at constant pressure. $100 \mathrm{~mL}$ toluene containing the catalyst precursor was transferred to the fully dried reactor under ethylene atmosphere. Then the required amount of cocatalyst $\left(\mathrm{AlEt}_{2} \mathrm{Cl}\right)$ was injected into the reactor using a syringe. As the temperature was reached $20^{\circ} \mathrm{C}$, the reactor was pressurized to prescribed pressure. After stirring for one hour, the reaction was quenched and worked up using the similar method described above for ordinary pressure reaction.

\section{Results and Discussion}

The NMR, element analysis, and IR characterization results of catalyst $\mathrm{NiL}_{2} \mathrm{Cl}_{2}$ and $\mathrm{NiL}_{1} \mathrm{Cl}_{2}$ are in well agreement with the literature [19], confirming the successful synthesis of homogenous diimine Cat.B and Cat.C. In the following part, the new material $\left[\mathrm{Zn}_{3}(\mathrm{OH})_{2}\left(\mathrm{~L}_{1}\right)_{2}\right](\mathrm{Zn}-\mathrm{MOF})$ will be characterized in detail.

\subsection{Characterization of the $\left[\mathrm{Zn}_{3}(\mathrm{OH})_{2}\left(L_{1}\right)_{2}\right]$}

3.1.1. XRD. XRD pattern of a synthesized Zn-MOF is displayed in Figure 1. The result showed that the XRD pattern of the synthesized sample was similar to that of a simulated pattern obtained from the single-crystal structure in the literature [21]. The crystallographic planes (110) and (220) of the sample have been denoted on the XRD patterns. However, there was a slight shift to lower angle region in the peak positions and the weakening of the peak intensities at higher angle region compared with those of the simulated one. The difference can possibly be caused by the unique 
TABLE 1: Effects of $n(\mathrm{Al}) / n(\mathrm{Ni})$ on the activity and distribution of oligomer ${ }^{\mathrm{a}}$.

\begin{tabular}{|c|c|c|c|c|c|c|c|c|c|}
\hline \multirow{2}{*}{ Entry } & \multirow{2}{*}{ Cat. } & \multirow{2}{*}{$n(\mathrm{Al}) / n(\mathrm{Ni})$} & \multicolumn{4}{|c|}{ Oligomer distribution $^{\mathrm{b}}$} & \multirow{2}{*}{$\alpha$-Olefin ${ }^{\mathrm{b}} / \%$} & \multirow{2}{*}{$\mathrm{Ao}^{\mathrm{c}}$} & \multirow{2}{*}{$A p^{c}$} \\
\hline & & & $\mathrm{C} 4 / \Sigma \mathrm{C}$ & $\mathrm{C} 6 / \Sigma \mathrm{C}$ & $\mathrm{C} 8 / \Sigma \mathrm{C}$ & $\geqq \mathrm{C} 10 / \Sigma \mathrm{C}$ & & & \\
\hline 1 & A & 200 & 29.8 & 15.4 & 24.3 & 30.5 & 30.7 & 0.9 & trace \\
\hline 2 & $\mathrm{~A}$ & 500 & 42.5 & 30.9 & 20.2 & 6.4 & 37.0 & 1.6 & 0.018 \\
\hline 3 & A & 1000 & 87.4 & 2.9 & 5.2 & 4.5 & 85.8 & 8.9 & trace \\
\hline 4 & $\mathrm{~A}$ & 1500 & 91.8 & 2.9 & 3.3 & 2.0 & 92.2 & 6.7 & trace \\
\hline 5 & B & 200 & 34.4 & 27.5 & 28.4 & 9.7 & 69.1 & 2.5 & trace \\
\hline 6 & B & 500 & 49.2 & 19.4 & 18.2 & 13.2 & 50.2 & 7.1 & trace \\
\hline 7 & $\mathrm{~B}$ & 1000 & 58.9 & 14.4 & 10.5 & 16.2 & 20.9 & 12.0 & trace \\
\hline 8 & B & 1500 & 72.4 & 14.1 & 4.5 & 9.0 & 15.0 & 8.2 & trace \\
\hline 9 & $\mathrm{C}$ & 200 & 29.9 & 16.6 & 15.2 & 38.3 & 24.9 & 4.7 & - \\
\hline 10 & C & 500 & 35.9 & 22.1 & 18.9 & 23.1 & 19.8 & 5.7 & - \\
\hline 11 & $\mathrm{C}$ & 1000 & 37.7 & 18.5 & 22.5 & 21.3 & 23.7 & 6.9 & - \\
\hline 12 & $\mathrm{C}$ & 1500 & 49.6 & 25.6 & 22.8 & 2.0 & 24.4 & 3.2 & - \\
\hline
\end{tabular}

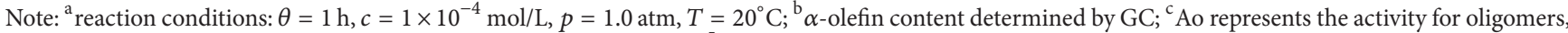
Ap is the activity for polymer, both activities have the same unit of $\times 10^{5} \mathrm{~g} /(\mathrm{mol} \cdot \mathrm{Ni} \cdot \mathrm{h} \cdot \mathrm{atm})$.

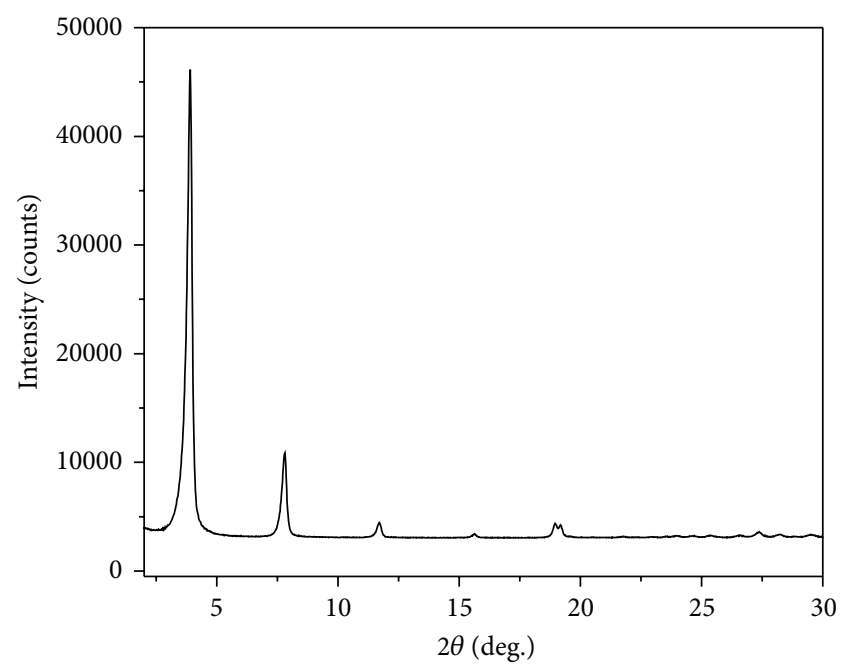

FIGURE 1: XRD pattern of Zn-MOF.

diimine ligands. As a result, it can also be concluded that the synthesized sample and the simulated one possess the similar porous structure.

3.1.2. SEM. As shown in Figure 2, the SEM images of $\left[\mathrm{Zn}_{3}(\mathrm{OH})_{2}\left(\mathrm{~L}_{1}\right)_{2}\right]$ present a regular flat stick crystal with average size of about $20 \mu \mathrm{m}$ in the length direction and $3 \mu \mathrm{m}$ in the width direction. Moreover, a small amount of irregular particles appears (Figure 2(a)), which could be unreacted ligands.

3.1.3. ICP. The elemental contents of samples were analyzed by the inductively coupled plasma (ICP) method. The analysis results showed that the content of elements $\mathrm{C}, \mathrm{H}$, and $\mathrm{N}$ over the Zn-MOF was $31.41,3.08$, and $4.12 \%$. Furthermore, the total Ni content of porous coordination polymer $\left[\mathrm{Zn}_{3}(\mathrm{OH})_{2}\left(\mathrm{~L}^{\mathrm{Ni}}\right)_{2}\right]_{n}$ was $11.39 \%$. Obviously, the content is apparently higher than those of traditional heterogeneous catalysts reported by some researchers before [22]. The results also suggested that the catalyst $\left[\mathrm{Zn}_{3}(\mathrm{OH})_{2}\left(\mathrm{~L}^{\mathrm{Ni}}\right)_{2}\right]_{n}($ Cat.A) had much more metal active sites for oligomerization catalysis compared to the other heterogeneous oligomerization catalysts. Much more metal active sites are helpful to improve the catalytic performance of ethylene oligomerization catalysts.

\subsection{Ethylene Oligomerization and Polymerization with $\mathrm{Et}_{2} \mathrm{AlCl}$ as Cocatalyst}

3.2.1. Effects of $n(\mathrm{Al}) / n(\mathrm{Ni})$ Values. In the present work, the catalytic activities of three catalysts (Cat.A, Cat.B, and Cat.C) were investigated. The catalytic test results were listed in Table 1.

As can be seen in Table 1, the optimal catalytic activities of all three catalysts were obtained when the $n(\mathrm{Al}) / n(\mathrm{Ni})$ value was $1000: 1$, respectively. As suggested in the literature [23], the surface of active species being overlaid with an excess amount of $\mathrm{Et}_{2} \mathrm{AlCl}$ can lead to the deactivation of active catalytic sites. It indicated that the proper $n(\mathrm{Al}) / n(\mathrm{Ni})$ value was the key factor for the catalytic performance of the catalyst. However, for the product distributions, it was surprising to observe that the content of $\mathrm{C} 4$ oligomers increases significantly with the increase of the $n(\mathrm{Al}) / n(\mathrm{Ni})$ values. In particular, for Cat.A, the content of $\mathrm{C} 4$ oligomers was obviously increased from $29.8 \%$ to $91.8 \%$. One reason for these results is that the chain transfer rate increased with the increase of $n(\mathrm{Al}) / n(\mathrm{Ni})$ values. Meanwhile, it also led to the decrease in the molecular weight of polyethylenes. In addition, the contents of $\mathrm{C} 4$ oligomers obtained over different catalysts were different; they followed the order: Cat.C $(49.6 \%)<$ Cat.B $(72.4 \%)$ < Cat.A $(91.8 \%)$. This is because nickel active sites locate in different electronic and special environments in three catalysts. The electron withdrawing inductive effect of carboxyl substituent groups attaching to the diimine ligands in Cat.A and Cat.B has positive effects 


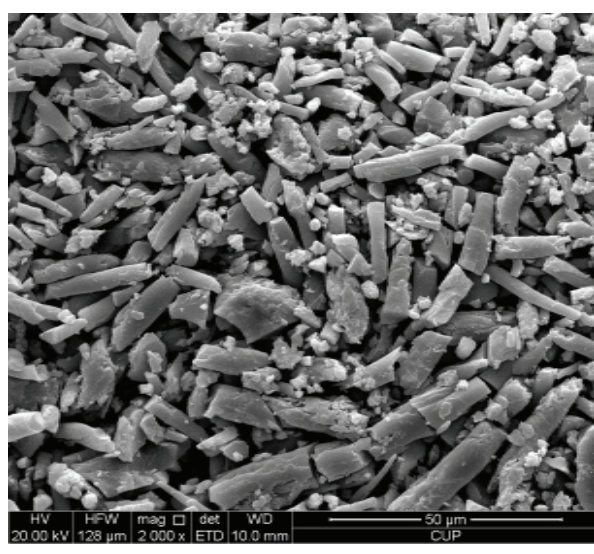

(a)

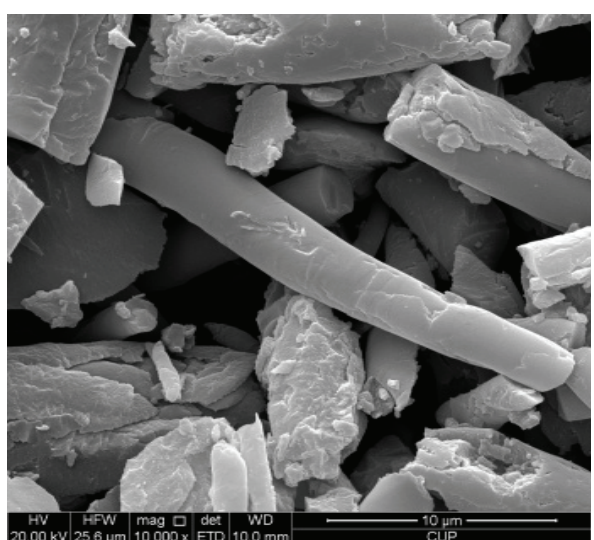

(b)

Figure 2: SEM images of Zn-MOF.

TABLE 2: Effects of temperature on the distribution of oligomer.

\begin{tabular}{|c|c|c|c|c|c|c|c|c|c|}
\hline \multirow{2}{*}{ Entry } & \multirow{2}{*}{ Cat. } & \multirow{2}{*}{$\mathrm{T} /{ }^{\circ} \mathrm{C}$} & \multicolumn{4}{|c|}{ Oligomer distribution } & \multirow{2}{*}{$\alpha$-Olefin $/ \%$} & \multirow{2}{*}{ Ao } & \multirow{2}{*}{ Ap } \\
\hline & & & $\mathrm{C} 4 / \Sigma \mathrm{C}$ & $\mathrm{C} 6 / \Sigma \mathrm{C}$ & $\mathrm{C} 8 / \Sigma \mathrm{C}$ & $\geqq \mathrm{C} 10 / \Sigma \mathrm{C}$ & & & \\
\hline 1 & $\mathrm{~A}$ & 0 & 29.8 & 30.9 & 34.8 & 4.5 & 37.0 & 0.9 & trace \\
\hline 2 & A & 20 & 87.4 & 2.9 & 5.2 & 4.5 & 85.8 & 8.9 & trace \\
\hline 3 & A & 40 & 39.9 & 26.1 & 26.8 & 7.2 & 55.3 & 1.1 & 0.02 \\
\hline 4 & A & 60 & 20.1 & 37.0 & 39.1 & 3.8 & 30.3 & 0.76 & trace \\
\hline 5 & B & 0 & 21.8 & 23.9 & 27.1 & 27.2 & 27.2 & 1.6 & trace \\
\hline 6 & B & 20 & 58.9 & 14.4 & 10.5 & 16.2 & 50.2 & 12.0 & trace \\
\hline 7 & B & 40 & 50.9 & 26.3 & 22.4 & 0.4 & 30.5 & 5.7 & trace \\
\hline 8 & B & 60 & 38.9 & 26.7 & 28.9 & 5.5 & 27.7 & 1.2 & - \\
\hline 9 & $\mathrm{C}$ & 0 & 59.4 & 6.1 & 9.5 & 25 & 28.1 & 10.9 & - \\
\hline 10 & $\mathrm{C}$ & 20 & 37.7 & 18.5 & 22.5 & 21.3 & 23.7 & 4.7 & - \\
\hline 11 & $\mathrm{C}$ & 40 & 35.9 & 14.3 & 11.6 & 38.2 & 43.9 & 2.9 & - \\
\hline 12 & $\mathrm{C}$ & 60 & 26.9 & 21.1 & 17.1 & 34.9 & 15.1 & 1.6 & - \\
\hline
\end{tabular}

Reaction conditions: $\theta=1 \mathrm{~h}, c=1 \times 10^{-4} \mathrm{~mol} / \mathrm{L}, n(\mathrm{Al}) / n(\mathrm{Ni})=1000, p=1.0 \mathrm{~atm}$.

for C4 formation. Apparently, Cat.A with self-supported nickel active sites possessed the best catalytic activity for $\mathrm{C} 4$ oligomer.

3.2.2. Effects of Reaction Temperature. As shown in Table 2, the result phenomena for the oligomerization activities of the catalysts with temperatures changing from 0 to $60^{\circ} \mathrm{C}$ were the same as those for the influences of $n(\mathrm{Al}) / n(\mathrm{Ni})$ values on the catalytic activities. The optimal activity of the catalyst was obtained at 20,20 , and $0^{\circ} \mathrm{C}$ for Cat.A, Cat.B, and Cat.C, respectively. And the reaction temperature was continued to increase and it resulted in the decreasing of catalytic activity. The reason might be caused by deactivation of some active centers and the lower concentration of ethylene in the reaction solution [24].

In addition, with the increasing of the reaction temperature, both catalytic reaction rate and the reversible $\beta$ - $\mathrm{H}$ elimination rate increase, which also led to the lower selectivity for C4 [25]. In our experiments, the major products are C4 and the minor products are $\mathrm{C} 6$ and $\mathrm{C} 8$ by using Cat.A and Cat.B, while the Cat.C also produced a large amount of C10. This phenomenon might be attributed to the carboxyl group, which led to stereo-hindrance effects.

3.2.3. Effects of Ethylene Pressure. The effects of pressure on the activities and distributions of oligomer and polymer are listed in Table 3. It can be seen that when the reaction pressure changed from 10 to 20 atm, for Cat.B and Cat.C, the catalytic activity of oligomerization increased. It attributed to the higher monomer concentration around active nickel centers at a proper higher pressure. However, further increasing reaction pressure resulted in lower catalytic activities. The reason was considered the saturation of ethylene and obstruction contacted with active species by polymer. Interestingly, the oligomerization activity of the Cat.A only varied between 9.15 and $11 \times 10^{5} \mathrm{~g} /(\mathrm{mol} \cdot \mathrm{Ni} \cdot \mathrm{h} \cdot \mathrm{atm})$. Its activity was less affected by pressures compared with those of other catalysts. The reason can be concluded that the heterogeneous catalyst Cat.A was 
TABLE 3: Effects of pressure on the distribution of oligomer.

\begin{tabular}{|c|c|c|c|c|c|c|c|c|c|}
\hline \multirow{2}{*}{ Entry } & \multirow{2}{*}{ Cat. } & \multirow{2}{*}{ p/atm } & \multicolumn{4}{|c|}{ Oligomer distribution } & \multirow{2}{*}{$\alpha$-Olefin/\% } & \multirow{2}{*}{ Ao } & \multirow{2}{*}{ Ap } \\
\hline & & & $\mathrm{C} 4 / \Sigma \mathrm{C}$ & $\mathrm{C} 6 / \Sigma \mathrm{C}$ & $\mathrm{C} 8 / \Sigma \mathrm{C}$ & $\geqq \mathrm{C} 10 / \Sigma \mathrm{C}$ & & & \\
\hline 1 & $\mathrm{~A}$ & 10 & 71.9 & 2.3 & 3.6 & 22.2 & 50.6 & 11.0 & 0.023 \\
\hline 2 & $\mathrm{~A}$ & 15 & 86.3 & 0.8 & 3.6 & 9.3 & 61.4 & 9.15 & 9.4 \\
\hline 3 & A & 20 & 86.4 & 5.6 & 5.3 & 2.7 & 71.7 & 10.8 & 0.24 \\
\hline 4 & $\mathrm{~A}$ & 25 & 87.6 & 6.0 & 4.6 & 1.8 & 72.3 & 9.25 & 0.025 \\
\hline 5 & B & 10 & 58.9 & 2.2 & 1.9 & 37.0 & 50.2 & 13.0 & 0.65 \\
\hline 6 & $\mathrm{~B}$ & 15 & 66.5 & 23.5 & 4.6 & 5.4 & 48.8 & 19.5 & 22.5 \\
\hline 7 & B & 20 & 93.0 & 3.2 & 2.4 & 1.4 & 60.4 & 24.0 & 34.0 \\
\hline 8 & B & 25 & 94.9 & 1.1 & 2.4 & 1.6 & 93.4 & 10.0 & 5.0 \\
\hline 9 & $\mathrm{C}$ & 10 & 39.7 & 25.9 & 24.1 & 10.3 & 31.7 & 16.0 & 0.19 \\
\hline 10 & $\mathrm{C}$ & 15 & 44.0 & 18.4 & 13.1 & 24.5 & 36.3 & 69.0 & 0.24 \\
\hline 11 & $\mathrm{C}$ & 20 & 49.0 & 20.2 & 11.2 & 19.6 & 51.4 & 108 & 0.38 \\
\hline 12 & $\mathrm{C}$ & 25 & 48.2 & 12.6 & 10.2 & 29.0 & 71.4 & 16.3 & 0.225 \\
\hline
\end{tabular}

Reaction conditions: $\theta=1 \mathrm{~h}, n(\mathrm{Al}) / n(\mathrm{Ni})=1000, T=20^{\circ} \mathrm{C}, c=1 \times 10^{-4} \mathrm{~mol} / \mathrm{L}$.

insoluble in the toluene. Moreover, $\mathrm{Ni}$ active sites were able to be effectively immobilized and to be evenly distributed on the metal-organic framework of the catalyst.

Comparing the catalytic activities over Cat.B and Cat.C, it is able to be found that the Cat.B with carboxyl groups in diimine ligands was inferior to the Cat.C without carboxyl groups in diimine ligands. However, the content of $\mathrm{C} 4$ compounds for the Cat.B had a distinct advantage over that for the Cat.C. The phenomena were due to the fact that the electron withdrawing effects of the carboxyl groups in Cat.B decreased the electron cloud density of nickel centers and thus increased its infinity with ethylene molecules. Compared with the results at $1 \mathrm{~atm}$, the higher pressure showed that the activity of polymer remarkably increased, especially for Cat.A and Cat.B. It can be explained that the propagation rate was faster than hydrogen elimination rate at higher pressure [26].

\section{Conclusions}

An original $\mathrm{Zn}-\mathrm{MOF}\left[\mathrm{Zn}_{3}(\mathrm{OH})_{2} \mathrm{~L}_{2}\right]$ was synthesized by the self-assembly of diimine ligands $\left(1,4\right.$-bis $\left(4-\mathrm{CO}_{2} \mathrm{HC}_{6} \mathrm{H}_{4}\right)-2,3$ dimethyl-1,4-diazabutadiene). In addition, the novel ethylene oligomerization catalyst $\left[\mathrm{Zn}_{3}(\mathrm{OH})_{2}\left(\mathrm{~L}_{1}{ }^{\mathrm{Ni}}\right)_{2}\right]$ was successfully prepared by a postsynthetic treatment method. The ICP characterization results demonstrated that this material possessed a higher Ni content than traditional heterogeneous catalysts. All catalysts showed the catalytic performance of ethylene oligomerization under various reaction conditions. Among the catalysts, the heterogeneous Cat.A exhibited the optimal selectivity for the low-carbon olefin C4. It was attributed to carboxyl electronic effects and space-confined channel effects. It is noteworthy that the Cat.B with dicarboxylic acid groups had the best activity obtained at $20^{\circ} \mathrm{C}$. The activity was higher than that of the Cat.C without dicarboxylic acid groups (obtained at $0^{\circ} \mathrm{C}$ ). Obviously, the system temperature had a distinct influence on the catalytic performances. Compared with Cat.C, the dicarboxylic acid groups of Cat.A and Cat.B played important roles in promoting ethylene polymers at high pressure. Furthermore, the system pressure also presented a significant effect on the activities of the catalysts.

\section{Conflict of Interests}

The authors declare that there is no conflict of interests regarding the publication of this paper.

\section{Acknowledgments}

The authors acknowledge the support of this work by the National Natural Science Foundation of China (Grant no. 51072230, U1162118, 21106123) and the Scientific Research Foundation of the Education Ministry for Returned Chinese Scholars and the Scientific Research Foundation of China University of Petroleum, Beijing (01JB0149).

\section{References}

[1] B. Cornils and W. A. Herrmann, Applied Homogeneous Catalysis with Organometallic Compounds, vol. 1, Wiley-VCH, Weinheim, Germany, 1996.

[2] H. A. Wittcoff, B. G. Reuben, and J. S. Plotkin, Industrial Organic Chemicals in Perspective, Wiley Interscience, New York, NY, USA, 1980.

[3] W. Keim, F. H. Kowaldt, R. Goddard, and C. Krüger, "Novel coordination of (Benzoylmethylene) triphenylphosphorane in a nickel oligomerization catalyst," in Angewandte Chemie International Edition, vol. 17, pp. 466-467, 6 edition, 1987.

[4] W. Keim, A. Behr, B. Limbacker, and C. Kruger, "Novel nickeloligomerization catalysts with arsenic-oxygen chelate ligands," Angewandte Chemie International Edition, vol. 22, no. 6, p. 503, 1983.

[5] M. Lallemand, O. A. Rusu, E. Dumitriu, A. Finiels, F. Fajula, and V. Hulea, "NiMCM-36 and NiMCM-22 catalysts for the ethylene oligomerization: effect of zeolite texture and nickel cations/acid sites ratio," Applied Catalysis A: General, vol. 338, no. 1-2, pp. 37-43, 2008. 
[6] V. Hulea and F. Fajula, "Ni-exchanged AlMCM-41-an efficient bifunctional catalyst for ethylene oligomerization," Journal of Catalysis, vol. 225, no. 1, pp. 213-222, 2004.

[7] R. J. Kuppler, D. J. Timmons, Q.-R. Fang et al., "Potential applications of metal-organic frameworks," Coordination Chemistry Reviews, vol. 253, no. 23-24, pp. 3042-3066, 2009.

[8] M. Dincǎ and J. R. Long, "Hydrogen storage in microporous metal-organic frameworks with exposed metal sites," Angewandte Chemie, vol. 47, no. 36, pp. 6766-6779, 2008.

[9] B. Chen, X. B. Zhao, A. Putkham et al., "Surface interactions and quantum kinetic molecular sieving for $\mathrm{H} 2$ and $\mathrm{D} 2$ adsorption on a mixed metal-organic framework material," Journal of the American Chemical Society, vol. 130, no. 20, pp. 6411-6423, 2008.

[10] J.-R. Li, R. J. Kuppler, and H.-C. Zhou, "Selective gas adsorption and separation in metal-organic frameworks," Chemical Society Reviews, vol. 38, no. 5, pp. 1477-1504, 2009.

[11] L. Q. Ma, C. Abney, and W. B. Lin, "Enantioselective catalysis with homochiral metal-organic frameworks," Chemical Society Reviews, vol. 38, no. 5, pp. 1248-1256, 2009.

[12] M. D. Allendorf, C. A. Bauer, R. K. Bhakta, and R. J. T. Houk, "Luminescent metal-organic frameworks," Chemical Society Reviews, vol. 38, no. 5, pp. 1330-1352, 2009.

[13] B. Chen, L. Wang, Y. Xiao et al., "A luminescent metal-organic framework with Lewis basic pyridyl sites for the sensing of metal ions," Angewandte Chemie, vol. 48, no. 3, pp. 500-503, 2009.

[14] P. Horcajada, C. Serre, G. Maurin et al., "Flexible porous metalorganic frameworks for a controlled drug delivery," Journal of the American Chemical Society, vol. 130, no. 21, pp. 6774-6780, 2008.

[15] K. M. L. Taylor-Pashow, J. D. Rocca, Z. Xie, S. Tran, and W. Lin, "Postsynthetic modifications of iron-carboxylate nanoscale metal-organic frameworks for imaging and drug delivery," Journal of the American Chemical Society, vol. 131, no. 40, pp. 14261-14263, 2009.

[16] K. Kyogoku, C. Yamada, Y. Suzuki et al., "Syntheses of metalorganic framework compounds containing Ni-bipyridyl complex for oligomerization of ethylene," Journal of the Japan Petroleum Institute, vol. 53, no. 5, pp. 308-312, 2010.

[17] J. Canivet, S. Aguado, Y. Schuurman, and D. Farrusseng, "MOFsupported selective ethylene dimerization single-site catalysts through one-pot postsynthetic modification," Journal of the American Chemical Society, vol. 135, no. 11, pp. 4195-4198, 2013.

[18] A. N. Mlinar, B. K. Keitz, D. Gygi, E. D. Bloch, J. R. Long, and A. T. Bell, "Selective propene oligomerization with nickel(II)based metal organic frameworks," ACS Catalysis, vol. 4, no. 3, pp. 717-721, 2014.

[19] B. P. Buffin and A. Kundu, "Synthesis, characterization, and crystal structure of platinum(II) and palladium(II) chlorides with an acidic $\alpha$-diimine ligand," Inorganic Chemistry Communications, vol. 6, no. 6, pp. 680-684, 2003.

[20] M. Helldörfer, J. Backhaus, W. Milius, and H. G. Alt, " $\alpha$ diimine)nickel(II) complexes containing chloro substituted ligands as catalyst precursors for the oligomerization and polymerization of ethylene," Journal of Molecular Catalysis A: Chemical, vol. 193, no. 1-2, pp. 59-70, 2003.

[21] R. Kitaura, G. Onoyama, H. Sakamoto, R. Matsuda, S.-I. Noro, and S. Kitagawa, "Immobilization of a metallo Schiff base into a microporous coordination polymer," Angewandte Chemie, vol. 43 , no. 20 , pp. $2684-2687,2004$.
[22] M. Lallemand, A. Finiels, F. Fajula, and V. Hulea, "Catalytic oligomerization of ethylene over Ni-containing dealuminated Y zeolites," Applied Catalysis A: General, vol. 301, no. 2, pp. 196201, 2006.

[23] S. Zhang, S. Y. Jie, Q. S. Shi, and W.-H. Sun, "Chromium(III) complexes bearing 2-imino-1,10-phenanthrolines: synthesis, molecular structures and ethylene oligomerization and polymerization," Journal of Molecular Catalysis A: Chemical, vol. 276, no. 1-2, pp. 174-183, 2007.

[24] S. Y. Jie, D. H. Zhang, T. Z. Zhang et al., "Bridged bispyridinylimino dinickel(II) complexes: syntheses, characterization, ethylene oligomerization and polymerization," Journal of Organometallic Chemistry, vol. 690, no. 7, pp. 1739-1749, 2005.

[25] R. Gao, M. Zhang, T. Liang, F. Wang, and W.-H. Sun, "Nickel(II) complexes chelated by 2-arylimino-6-benzoxazolylpyridine: syntheses, characterization, and ethylene oligomerization," Organometallics, vol. 27, no. 21, pp. 5641-5648, 2008.

[26] W. J. Zhang, W.-H. Sun, S. Zhang et al., "Synthesis, characterization, and ethylene oligomerization and polymerization of [2,6bis(2-benzimidazolyl)pyridyl]chromium chlorides," Organometallics, vol. 25, no. 8, pp. 1961-1969, 2006. 

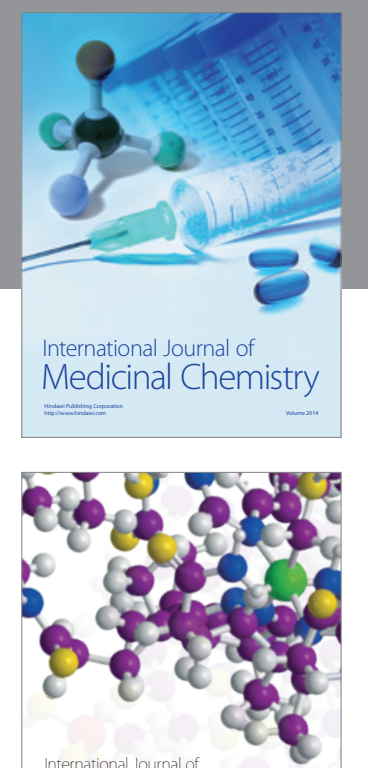

\section{Carbohydrate} Chemistry

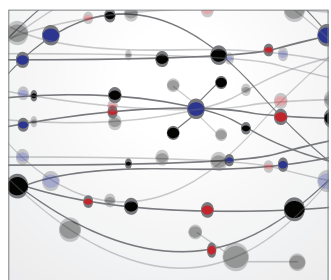

The Scientific World Journal
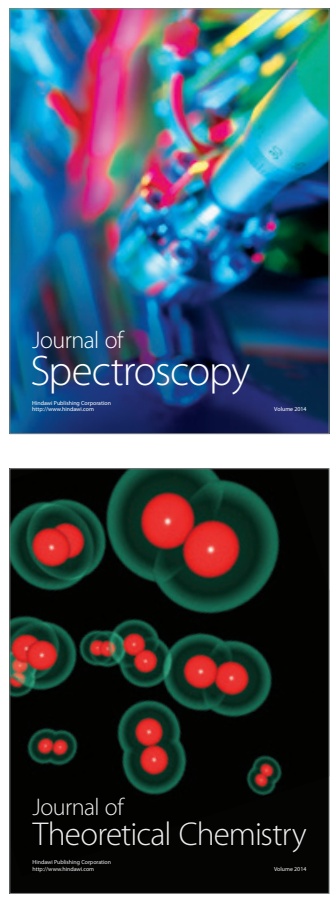
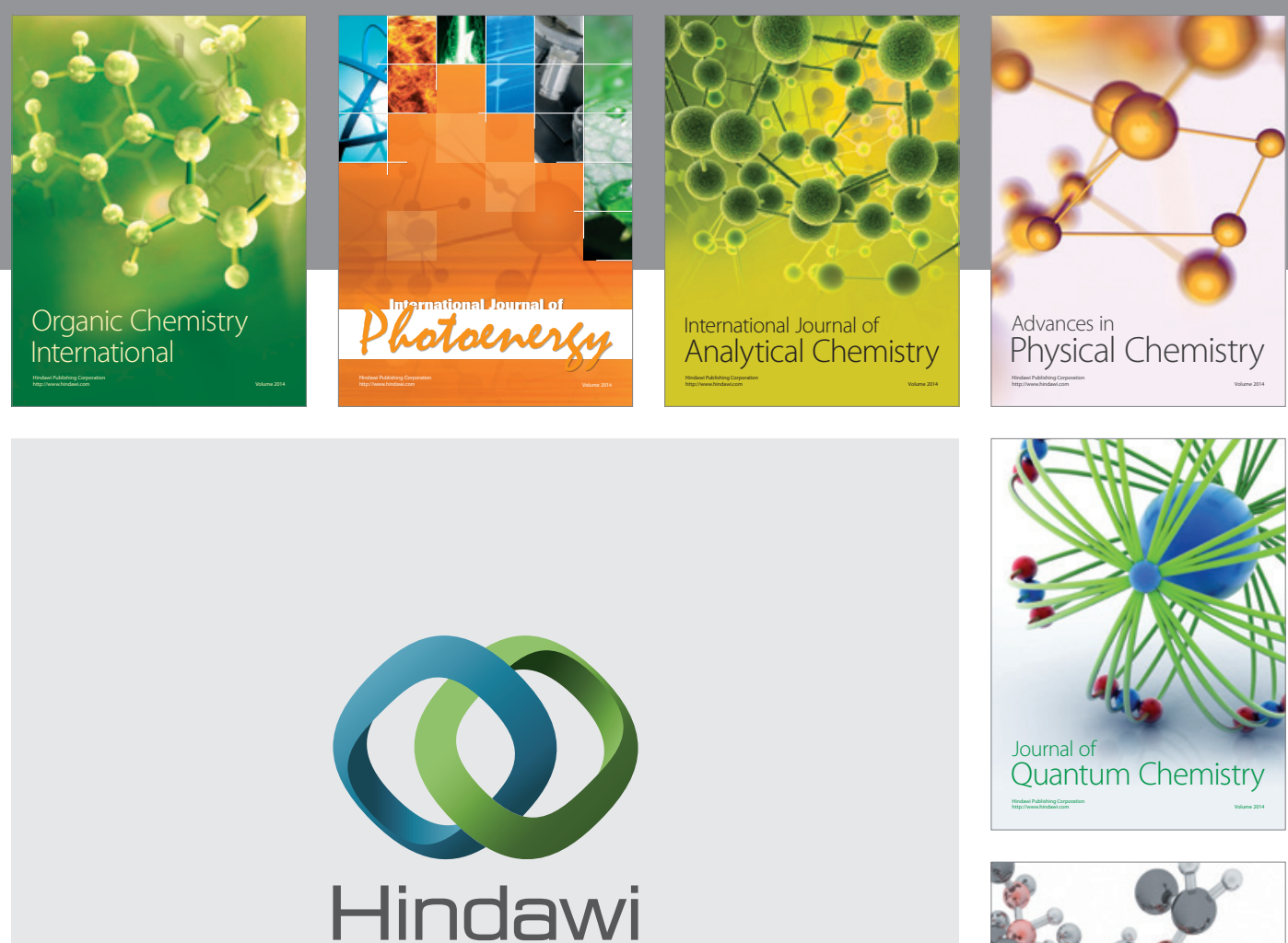

Submit your manuscripts at

http://www.hindawi.com

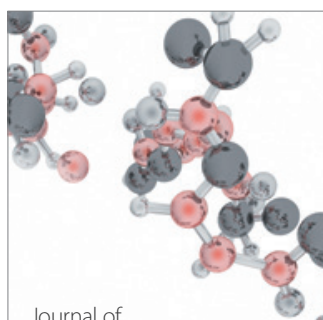

Analytical Methods

in Chemistry

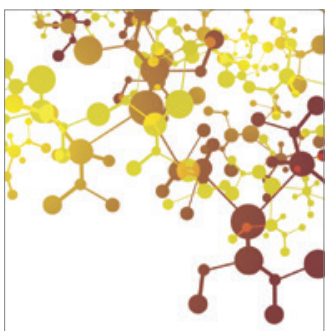

Journal of

Applied Chemistry

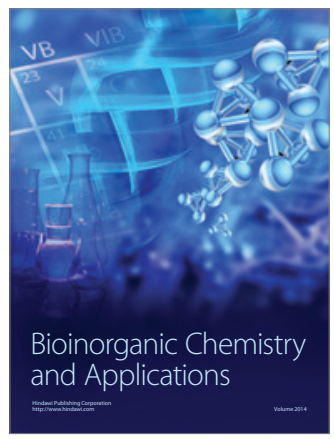

Inorganic Chemistry
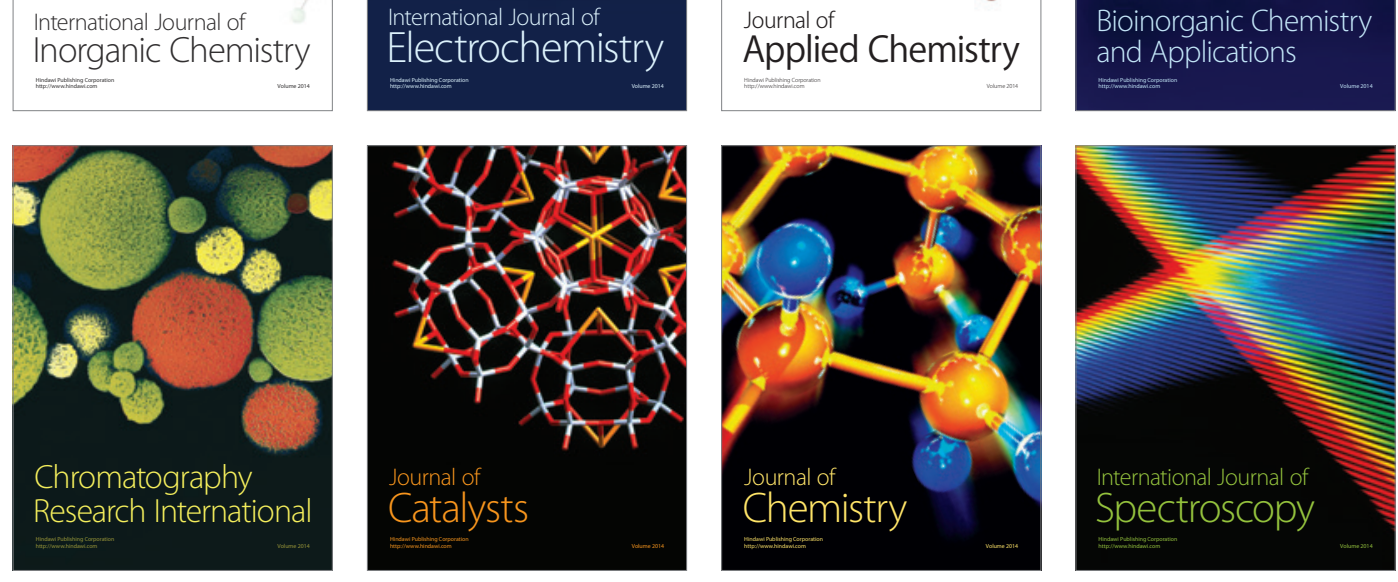